\title{
Digital n- $\gamma$ Pulse Shape Discrimination in Organic Scintillators with a High-Speed Digitizer
}

\author{
Chanho Kim', Jung-Yeol Yeom', Geehyun Kim²,* \\ ${ }^{1}$ Department of Bioengineering, Korea University, Seoul, Korea; ${ }^{2}$ Department of Nuclear Engineering, Sejong University, Seoul, Korea
}

\section{Original Research}

Received October 11, 2018

Revision February 15, 2019

Accepted April 9, 2019

Corresponding author: Geehyun Kim

Department of Nuclear Engineering, Sejong University, 209 Neungdong-ro, Gwangjin-gu, Seoul 05006, Korea

Tel: +82-2-3408-4013

Fax: +82-2-3408-3182

E-mail:gkim01@sejong.ac.kr

\begin{abstract}
This is an Open-Access article distributed under the terms of the Creative Commons Attribution NonCommercial License (http://creativecommons.org/ licenses/by-nc/4.0) which permits unrestricted noncommercial use, distribution, and reproduction in any medium, provided the original work is properly cited.
\end{abstract}

Copyright $\odot$ 2019 The Korean Association for Radiation Protection

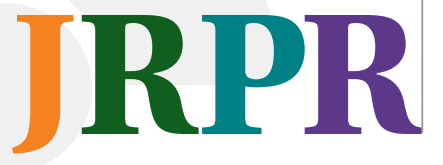

Background: As neutron fields are always accompanied by gamma rays, it is essential to distinguish neutrons from gamma rays in the detection of neutrons. Neutrons and gamma rays can be separated by pulse shape discrimination (PSD) methods. Recently, we performed characterization of a stilbene scintillator detector and an EJ-301 liquid scintillator detector with a highspeed digitizer DT5730 and investigated optimized PSD variables for both detectors. This study is for providing a basis for developing fast neutron/gamma-ray dual-particle imager.

Materials and Methods: We conducted PSD experiments using stilbene scintillator and EJ301 liquid scintillator and evaluated neutron and gamma ray discriminability of each PSD method with a ${ }^{137} \mathrm{Cs}$ gamma source and $\mathrm{a}^{252} \mathrm{Cf}$ neutron source. We implemented digital signal processing techniques to apply two PSD methods - the charge comparison (CC) method and the constant time discrimination (CTD) method - to distinguish neutrons from gamma rays. We tried to find optimized PSD variables giving the best discriminability in a given experimental condition.

Results and Discussion: For the stilbene scintillator detector, the charge comparison method and the constant time discrimination method both delivered the PSD FOM values of 1.7. For the EJ-301 liquid scintillator detector, both PSD methods delivered the PSD FOM values of 1.79. With the same PSD variables, PSD performance was excellent in $300 \pm 100 \mathrm{keVee}, 500 \pm 100 \mathrm{keVee}$, and $700 \pm 100$ keVee energy regions. This result shows that we can achieve an effective discrimination of neutrons from gamma rays using these scintillator detector systems.

Conclusion: We applied both PSD methods to a stilbene and a liquid scintillator and optimized the PSD performance represented by FOM values. We observed a good separation performance of both scintillators combined with a high-speed digitizer and digital PSD. These results will provide reference values for the dual-particle imager we are developing, which can image both fast neutrons and gamma rays simultaneously.

Keywords: Pulse shape discrimination, Stilbene scintillator, EJ-301 liquid scintillator, Digital signal processing, Dual-particle imager

\section{Introduction}

Detecting neutron essentializes discriminating neutron from background gamma ray because gamma radiation is also emitted by excited nuclei which produce neutrons [1]. For a certain type of radiation detectors, the pulse signal associated with gamma rays and neutrons exhibits slightly different shape depending on the type of incident radiation. Generally, organic scintillators such as stilbene and liquid scintillator have been used for detecting fast neutrons. They are known for excellent pulse shape dis- 
crimination (PSD) capability for identification of neutron from gamma ray. Stilbene scintillators have achieved widespread popularity as organic scintillators. Since the discovery of a stilbene scintillator in 1959, stilbene is preferred in those situations in which pulse shape electrons [1]. Stilbene scintillators have been a popular tool for much of the PSD experimental work, and previous studies have comprehensively proved that the stilbene scintillator has an excellent PSD capability $[2,3]$. However, stilbene traditionally grows with Bridgman technology, making it difficult to obtain in large sizes. This method has difficulties in limiting the size of the scintillator, because of stresses and cracks during growth and cooling [4]. Therefore, when a large single crystal is required, the stilbene scintillator is relatively high cost and the size of the stilbene scintillator is limited, making it difficult to utilize for a variety of applications. Solution-based stilbene scintillators were developed in 2013 by the Lawrence Livermore National Laboratory (LLNL). Solution-based stilbene scintillators can grow up to $10 \mathrm{~cm}$ in diameter. This scintillator, made with solution-based growth methods, is larger than Bridgman technique's one and improves light yield from incident radiation [4]. Recently, the solution-based stilbene scintillator has become commercially available, and neutron measurement studies with PSD using the solution-based stilbene scintillator have been extensively revisited. EJ-301 liquid scintillator (which is similar to NE-213, BC-501) also provides excellent PSD capability allowing for identification of neutron from gamma rays and can easily be made to large dimensions. For many years, EJ-301 liquid scintillator has been used for PSD research, and detailed characterization of EJ-301 PSD properties has been studied [5-7]. For organic scintillators such as stilbene and liquid scintillators, their response to electrons is almost linear for energies of incident particles above about $125 \mathrm{keV}$. However, their response to heavy charged particles such as protons is not linear but always less than the response to electrons of equivalent energies. Owing to the dependence of the light yield of organic scintillators on the type of particle, a special unit was duly introduced to describe the absolute light yield. The term keV electron equivalent (keVee) is, thus, used to represent the light yield on an absolute basis [1].

Analog electronic PSD systems have been conventionally used to obtain $\mathrm{n}-\gamma$ discrimination from the comparison of each pulse shape. These analog PSD systems have limited output count rate capability up to $\sim 200 \mathrm{kHz}$ [8]. They can only count the number of events classified as neutrons or gamma rays on-the-fly, while post-processing of the signal data is impossible after measuring radiation. Analog PSD systems, which have been used for a few recent decades, have started to be replaced by digital PSD methods using digital pulse processing (DPP) technology as high-speed digitizers have been developed. The first attempt to discriminate neutron and gamma ray digital pulses with NE-213 was performed in the 1980s. Two channels of the analog-to-digital converter (ADC) were used for analysis fast and slow decay components [9]. The digital PSD method with DPP technique has several advantages over analog electronic PSD systems. With digital PSD, one can either analyze the detector signal in real time using the FPGA, or store information, such as signal amplitude and time, in the PC for further analysis. As it usually requires a high-speed digitizer only, the detection system configuration becomes relatively simpler and lighter.

Recently, we obtained a commercially available solutionbased stilbene crystal from Inrad Optics, an EJ-301 liquid scintillator from ELJEN TECHNOLOGY and a high-speed digitizer DT5730 (14-bit, 500 MS/s) from CAEN. Depending on the time width of typical pulse signals being processed, one can achieve processible count rate of pulses up to 500 $\mathrm{kHz}$, which exhibits an advantage over previous analog PSD systems. As a fundamental step for the development of a fast neutron/gamma-ray dual-particle imager [10-12], we took an initiative approach to implement a fast neutron/gammaray dual-particle measurement system with digital PSD using the stilbene crystal from Inrad Optics, and the EJ-301 liquid scintillator from ELJEN TECHNOLOGY combined with DT5730 of CAEN. In the development of the fast neutron/ gamma-ray dual-particle imager, we take a single non-position-sensitive detector-based approach to obtain temporal modulation patterns determined by the rotating collimators. In order to handle the complex process of controlling the collimator rotation and acquiring measurement data according to the collimator rotation, applying PSD techniques, we had to develop our own customized code system to implement the PSD technique and the equipment control.

We performed an initial characterization for this system to provide information for the future implementation of the neutron/gamma-ray dual-particle imager not only for the dual-particle imager, but also in various studies and applications. Neutrons and gamma rays were measured with two types of organic scintillators combined with a high-speed digitizer, at Korea Institute of Nuclear Nonproliferation and 
Control (KINAC). Two types of digital PSD algorithm were applied for discriminating between neutrons and gamma rays, which are the charge comparison (CC) method and the constant time discrimination (CTD) method. And we focused on finding an optimized parameter for two digital PSD methods respectively.

\section{Experimental Setup}

A solution-based stilbene scintillator ( 2 in. $\times 2$ in., Inrad Optics) and an EJ-301 liquid scintillator ( 2 in. $\times 2$ in., ELJEN TECHNOLOGY) were used for detecting neutrons and gamma rays. The stilbene scintillator was coupled to a H6525 PMT ( 3 in. diameter, HAMAMATSU) and EJ-301 detector was coupled to a R7724 PMT (2 in. diameter, HAMAMATSU). The H6525 PMT was operating at $-1,400 \mathrm{~V}$ and the R7724 PMT was operating at $-1,050 \mathrm{~V}$. The anode signal of PMT was sent directly to a high-speed digitizer: DT5730 (500 MS/s, 14-bit resolution, CAEN). Throughout the experiment, the sampling rate of DT5730 digitizer set to $500 \mathrm{MS} / \mathrm{s}$, therefore, the interval between each sample corresponds to 2 ns. For data analysis, data was stored in PC, and the PSD parameter was calculated with the Matlab ${ }^{\circledR}$ software. A standard disk type ${ }^{137} \mathrm{Cs}$ source was used as a gamma-ray source and a capsule type ${ }^{252} \mathrm{Cf}$ source was used as a fast neutron source to investigate the PSD response of the detector. Detailed information of radiation sources is shown in Table 1.

As shown in Figure 1, the radiation source was located $25 \mathrm{~cm}$ away from the detector. Each source was measured for

Table 1. List of Radiation Sources Used in the Experiment

\begin{tabular}{lllc}
\hline Isotope & Source type & Activity & Reference date \\
\hline${ }^{137} \mathrm{Cs}$ (661.67 keV, Gamma ray) & Disc type & $50 \mu \mathrm{Ci}$ & 2008. 09. 01 \\
${ }^{252} \mathrm{Cf}$ (Neutron and gamma ray) & Capsule type & $90 \mu \mathrm{Ci}$ & 2015. 10. 15 \\
\hline
\end{tabular}

\section{0 minutes.}

In this experiment setup, the output signal of PMT is connected to the DT5730 digitizer without a charge sensitive preamplifier or a shaping amplifier. So, deposited energy of an incident particle associated with each pulse corresponds to the sum of $\mathrm{ADC}$ channel values in each pulse, and we call it as an 'integrated charge' of pulse. We used the ${ }^{137} \mathrm{Cs}$ source to calibrate the integrated charge of stilbene scintillator and EJ-301 liquid scintillator pulses to the deposited energy. Energy calibration was performed assuming Compton edge energy of $477.34 \mathrm{keVee}$ at $80 \%$ height of edge maximum [13]. Figure 2 shows spectra of integrated charge per each pulse created on the stilbene and EJ-301 liquid scintillators using the ${ }^{137} \mathrm{Cs}$ source for the calibration measurement. In the integrated charge pulse spectrum of ${ }^{137} \mathrm{Cs}$, integrated charge values of 15,645 and 12,865 pulses correspond to the 477.34 keVee Compton edge of 661.67 keVee for the EJ-301 liquid scintillator and the stilbene scintillator, respectively. As dif-

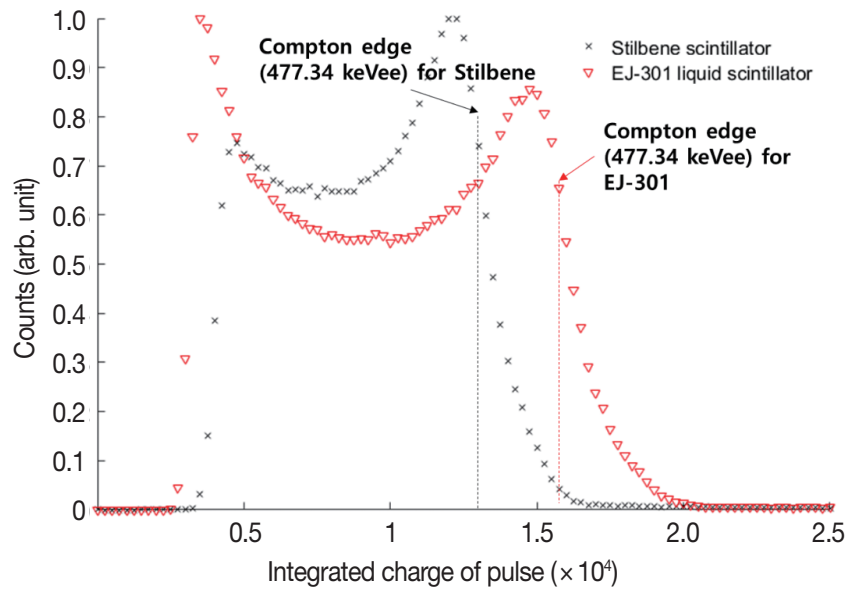

Fig. 2. Energy-channel calibration of the stilbene scintillator and the EJ-301 liquid scintillator using ${ }^{137} \mathrm{Cs}$.
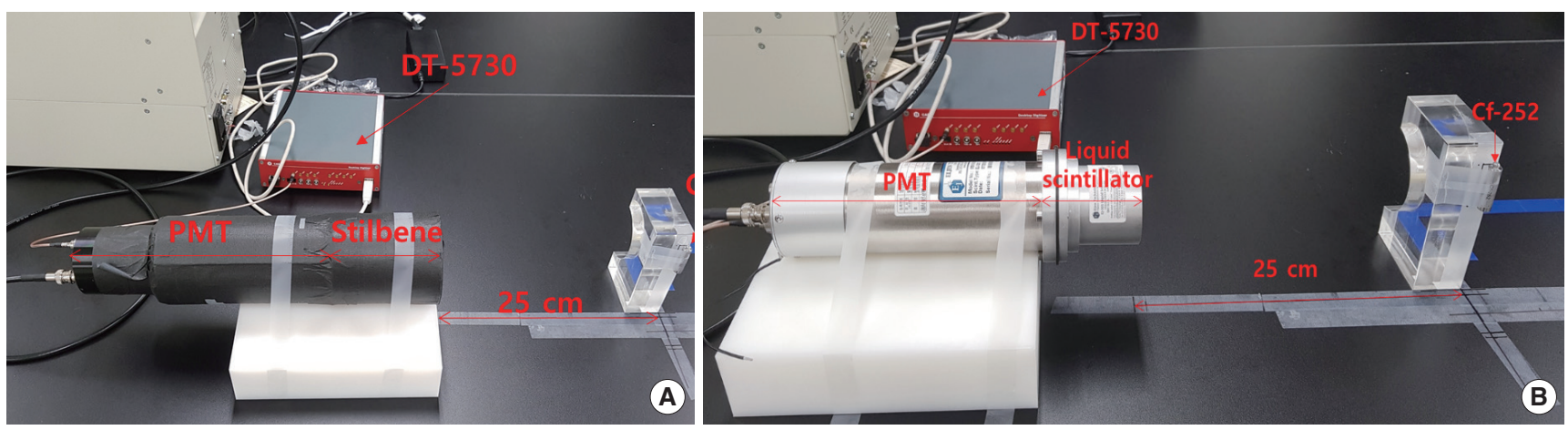

Fig. 1. Configurations of neutron/gamma-ray measurement experiment using a stillbene scintillator detector (A) and an EJ-301 liquid scintillator (B). 
ferent types of PMTs were connected to the EJ-301 liquid scintillator and stilbene scintillator, relative light yields of two scintillators were hard to compare with these data only.

\section{Materials and Methods}

In organic scintillators, the decay time of fluorescence depends on the type of incoming particles. When radiation particles are incident on the organic scintillator, radiation particles mainly excite $\pi$-electrons, and they are raised to singlet states $\left(\mathrm{S}_{\mathrm{s}}\right)$ or triplet states $\left(\mathrm{S}_{\mathrm{T}}\right)$. Prompt fluorescence, which is known as the fast component of the scintillation process, is emitted when excited $\pi$-electrons in the $S_{s}$ state return to the ground state. The lifetime of the $S_{s}$, which is measured in nanoseconds, is shorter than that of the $\mathrm{S}_{\mathrm{T}}$ which can be up to 1 millisecond [14]. The delayed fluorescence, which is known as the slow component of the scintillation process, is emitted when the particle in the $\mathrm{S}_{\mathrm{T}}$ state transferring to the $S_{s}$ before returning to the ground state. Therefore, fluorescence emission is delayed. At low excitation densities, almost no interaction between excited molecules occurs, so all singlet excitation decay rapidly. In contrast, the triplet annihilation is bimolecular and therefore depends on excitation density, so delayed fluorescence, originating from triplet annihilation, is observed under high excitation density. For incident particles of high energy-loss rate in a medium, one can expect to see increased excitation density of electrons at vicinity, so can one for the triplet state density [2]. In scintillators, heavy charged particles created by neutron capture reactions and recoil protons generated by elastic scatterings of neutrons, exhibit much greater ener- gy-loss rates than primary and secondary electrons generated by gamma rays. Therefore, one can observe relatively higher density of triplet states and, thus, an increased fraction of delayed fluorescence from the neutron interaction in organic scintillators [15].

Some organic scintillators, including stilbene scintillators and a few commercial liquid scintillators, offer excellent PSD performances because slow components of the light emission will appear differently depending on the incident radiation particles $[1,16]$. Therefore, we can use various PSD methods utilizing the difference between decay times of gamma-ray and neutron pulses from the stilbene scintillator and the EJ-301 liquid scintillator. Two types of PSD algorithms were implemented on off-line analysis using a Mat$\mathrm{lab}^{\circledR}$ code to calculate PSD parameters for each signal. Two types of PSD algorithm, are the charge comparison method and the constant time discrimination method, as described below in detail.

\section{Charge comparison (CC) method}

First, we performed the digital PSD using the charge comparison method. As mentioned above, the neutron pulse has a longer decay time than that of gamma-ray pulses because the recoil proton, generated by the neutron elastic scattering, provokes longer delayed emissions from the scintillator than electrons from gamma ray interactions do. Therefore, the CC method makes use of the difference in the fraction of delayed fluorescence between the signal generated by the neutron and the signal generated by the gamma ray as shown in Figure 3. This method uses the ratio of $Q_{\text {slow }}$ to $Q_{\text {long }}$ as a PSD parameter. In here, $Q_{\text {long }}$ is integrated charge of the total signal

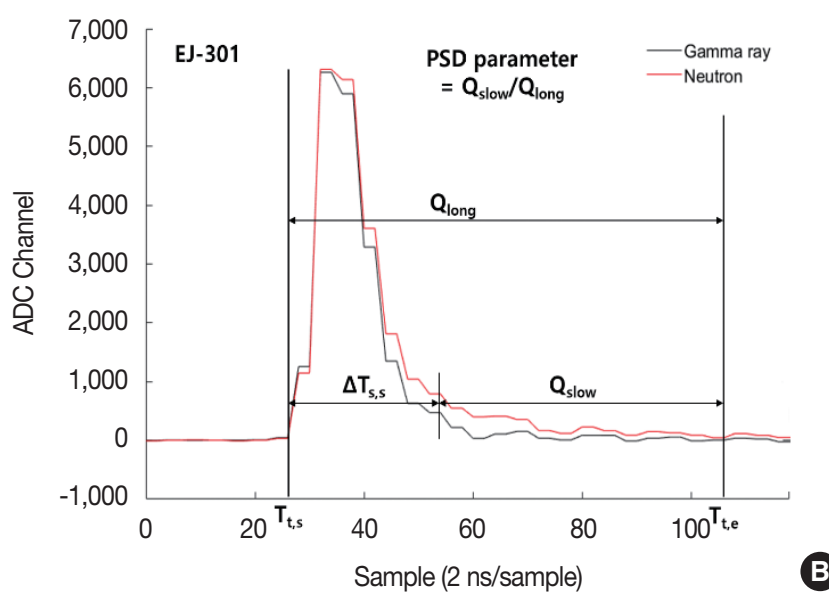

Fig. 3. Schematics of charge comparison method implementation on neutron and gamma signals from the stilbene (A) and the EJ-301 liquid scintillator (B) detectors. 
and $Q_{\text {slow }}$ is integrated charge from a specific beginning point to end of radiation signal.

The PSD parameter in the CC method can be defined as follows:

$$
P S D \text { parameter }=\frac{Q_{\text {slow }}}{Q_{\text {long }}}=\frac{\int_{T_{t, s}+\Delta T_{s, S} Q d t}^{T_{t, e}}}{\int_{T_{t, S}}^{T_{t, e}} Q d t}
$$

Where $T_{t, s}$ and $T_{t, e}$ are the beginning and the end point of the integral range used for the integration charge of $Q_{\text {long, }}$ while $\Delta T_{s, s}$ is the time interval between the beginning point of radiation signal and beginning point of integral range used for the integration charge of $Q_{\text {slow }} . \Delta T_{s, s}$ is one of the most important factors affecting the performance of the PSD in the CC method, and $T_{t, s}$ and $T_{t, e}$ will also affect the performance of PSD in the CC method.

\section{Constant time discrimination (CTD)}

We also performed the digital PSD using the CTD method. This method is based on the shape difference of integrated pulses which is normalized to the full integral value of the
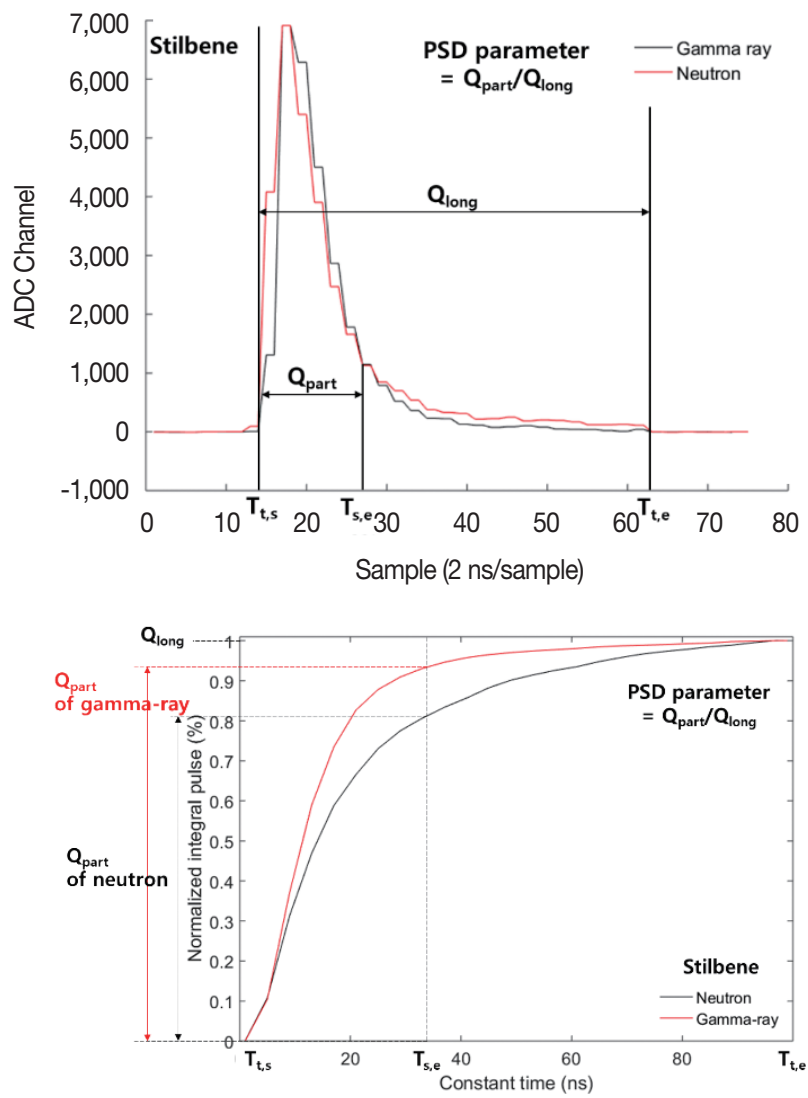

whole signal. As shown in Figure 4, we integrated the raw pulse signal obtained by the digitizer with 2 ns interval, for a certain amount of time period (slightly longer than typical pulse width) and normalized the integral pulse to the maximum value of each signal, i.e. the full integral value. As mentioned above, since the decay time of the neutron pulse is longer than the gamma ray pulse, the PSD parameter value of the normalized integral neutron pulse is to be smaller than that of the normalized integral gamma-ray pulse at a constant time. Here, the PSD parameter in the CTD method can be defined as follows (Figure 4):

$$
P S D \text { parameter }=\frac{Q_{\text {part }}}{Q_{\text {long }}}=\frac{\int_{T t, s}^{T_{s, e}} Q d t}{\int_{T_{t, s}}^{T_{t, e}} Q d t}
$$

Where $T_{s, e}$ is the time after a constant time from $T_{t, s}$, therefore the amplitude of the integral pulse at $T_{s, e}$ normalized to the full amplitude pulse is defined as the PSD parameter. In this respect, $T_{s, e}$ is one of the most important factors affecting the performance of the PSD in this method. In addition, $T_{t, s}$ and $T_{t, e}$ can also affect the performance of PSD in the CTD method.
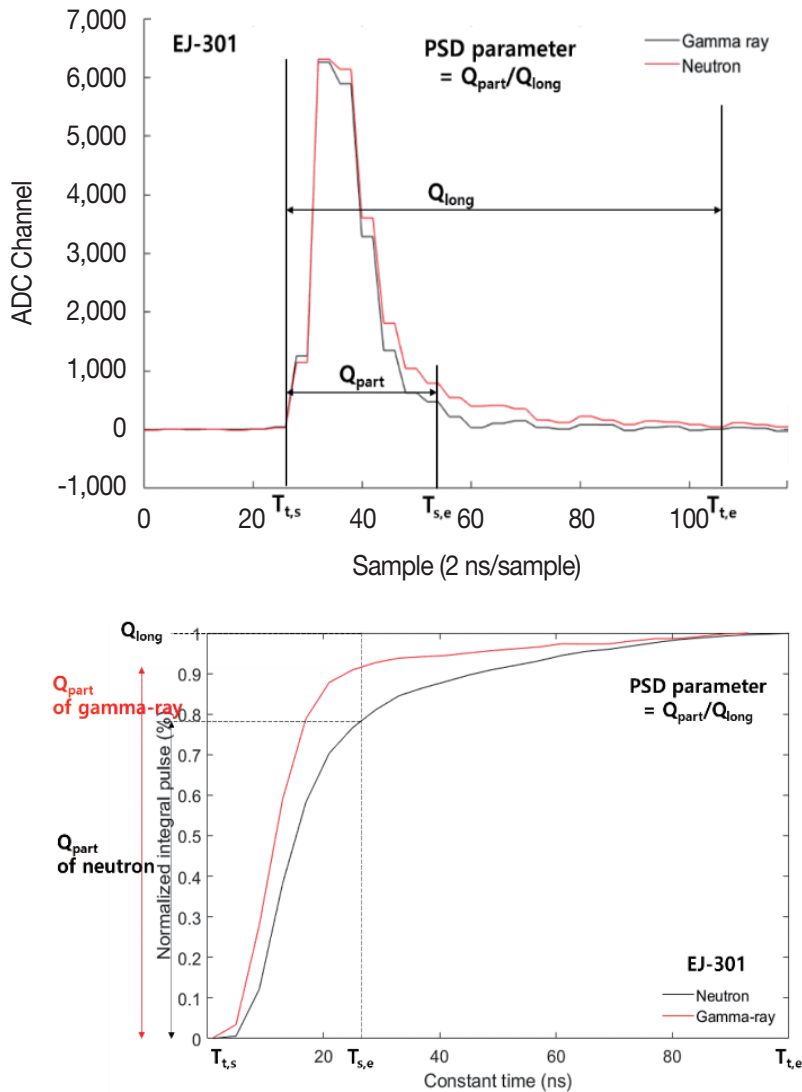

Fig. 4. Schematics of constant time discrimination method implementation on neutron and gamma signals from the stillbene (A) and the EJ301 liquid scintillator (B) detectors. Upper figures illustrate raw signals as-captured from the digitizer, whereas lower figures show integrated pulses of the raw signals for PSD application and normalized for illustration. 


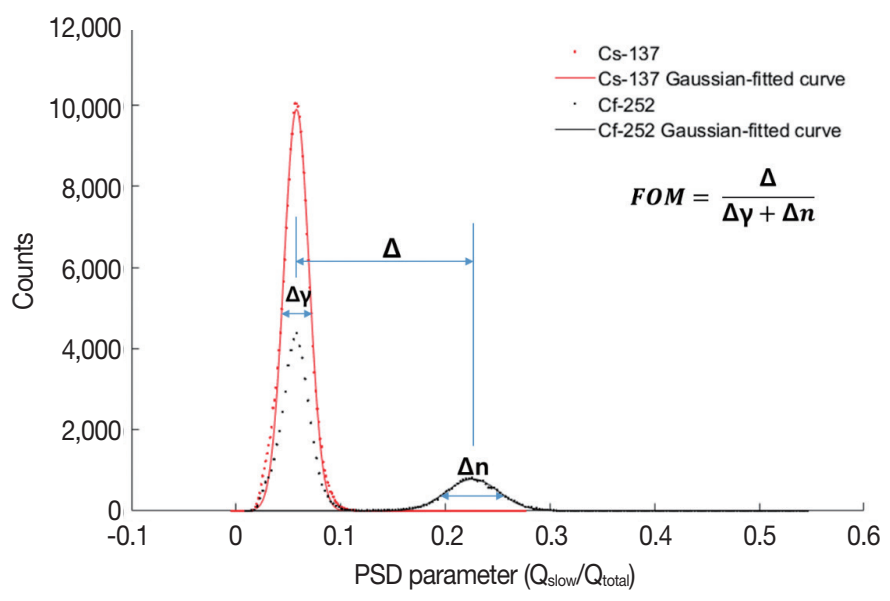

Fig. 5. The distribution of PSD parameters in CC method and the definition of FOM.

\section{Figure of Merit (FOM)}

The FOM can evaluate the separation performance of the PSD technique. The FOM can be obtained by plotting a distribution of the PSD parameter. FOM values are defined as $\mathrm{FOM}=\Delta /(\Delta \gamma+\Delta n)$, where $\Delta$ is the distance between peak centroids of each PSD parameter distribution, while $\Delta \gamma$ and $\Delta n$ are full width at half maximum (FWHM) values of gamma and neutron PSD parameter distributions, respectively, as shown in Figure 5. Therefore, the higher the FOM value, the greater the distance between neutrons and gamma-ray PSD parameter distribution, indicating that the PSD capability is more optimized.

To calculate the FOM value, both neutron and gamma-ray peaks of PSD parameter distribution were fitted with Gaussian-shaped curves. In principle, two particles are considered to be completely distinguished from each other, if the FOM value is greater than 1.5 [17].

\section{PSD parameter optimization}

As an attempt to maximize the FOM value, we calculated FOM values while changing thresholds for starting time $\left(T_{t, s}\right)$ and stopping time $\left(T_{t, e}\right)$ in both methods, the time interval $\left(\Delta T_{s, s}\right)$ for the CC method and the fractionating instance $\left(T_{s, e}\right)$ after $T_{t, s}$ in the CTD method. In case of the stilbene scintillator, the time interval $\left(\Delta T_{s, s}\right)$ and the fractionating instance $\left(T_{s, e}\right)$ were varied from $20 \mathrm{~ns}$ to $42 \mathrm{~ns}$, increasing by $2 \mathrm{~ns}$. The threshold was varied from $1 \%$ to $2.5 \%$ of the maximum amplitude of pulses, increasing by $0.5 \%$. In case of the EJ-301 liquid scintillator, those were changed from $16 \mathrm{~ns}$ to $32 \mathrm{~ns}$ with 2 ns steps and the threshold from $0.1 \%$ to $1.5 \%$ with $0.5 \%$ step. Considering the average pulse width of the scintillators being 44 ns for stilbene and 34 ns for EJ-301 at overall energy range, $\Delta T_{s, s}$ in CC and $T_{s, e}$ in CTD over 44 ns for stilbene and 34 ns for EJ-301 will become less meaningful because it will already cover the full width of the pulse after this.

\section{Discussion and Results}

Figure of merit (FOM) results for both scintillators using CC and CTD PSD methods are illustrated in Figure 6. When thresholds for $T_{t, s}$ and $T_{t, e}$ were varied, thresholds of $1.5 \%$ and $0.5 \%$ of the maximum amplitude showed good performances for the stilbene scintillator and the EJ-301 liquid scintillator, respectively. Optimal thresholds for the stilbene scintillator (Figure 6A and 6B) turned out to be higher than that of the EJ-301 liquid scintillator (Figure 6C and 6D).

The difference between optimal thresholds of the two scintillation detectors is speculated to have relationship with the light output difference between the two scintillators. The light yield of the stilbene scintillator is known to be about 1.76 times greater than the EJ-301 liquid scintillator $[18,19]$. However, the H6525 PMT, which is connected to the stilbene scintillator, shows a gain of about $3 \times 10^{4}$ when it is operating at $-1,400 \mathrm{~V}$ [19] and the R7724 PMT, which is connected to the EJ-301 liquid scintillator, shows a gain of about $1 \times 10^{5}$ when -1,050 V was applied for signal acquisition [19]. Therefore, the signal output of the EJ-301 liquid scintillator with the R7724 PMT is expected to be larger than that of the stilbene scintillator with the H6525 PMT for the same radiation energy. In addition, the maximum amplitude of the integrated charge signal will become larger for the scintillator with shorter decay time, as the total integrated charge value of the signal will be conserved in proportion with the light output from the radiation energy deposition. Mean decay times of first 3 components of light output are 4.3 ns, 34.8 ns, 332 ns for the stilbene scintillator [20] and are $3.16 \mathrm{~ns}, 32.3 \mathrm{~ns}, 270$ ns for the EJ-301 liquid scintillator [18]. The prompt portion of the decay time is shorter for the EJ-301 liquid scintillator, and the EJ-301 liquid scintillator detector will tend to exhibit larger amplitude signals compared to the stilbene scintillator detector providing the same gain settings for the same total light output. This also accounts for the typical pulse width results on both scintillators, where stilbene detector tends to show longer pulse widths. As shown in legends of Figure 6, optimal threshold (relative to the maximum amplitude represented in \%) of the stilbene scintillator detector tends to be higher than that of the EJ-301 liquid scintillator detector. 

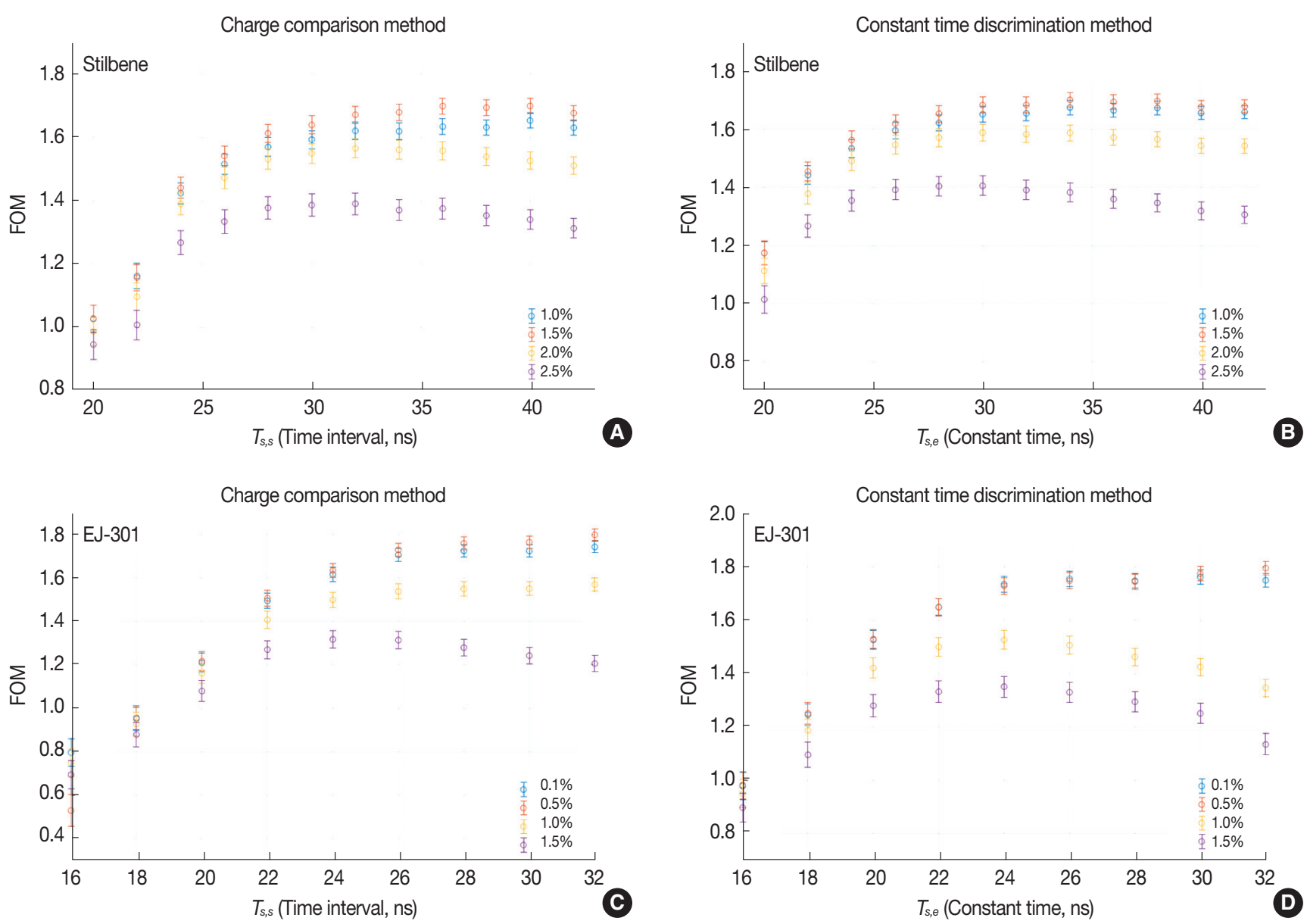

Fig. 6. At the full energy range, FOM values calculated for (A) the CC method and (B) the CTD method of the stillbene scintillator and (C) the CC method and (D) the CTD method of the EJ-301 liquid scintillator applying various time parameter $\left(\Delta T_{s, s}\right.$ and $\left.T_{s, e}\right)$ and threshold conditions.

Figure 6 also shows the FOM value depending on $\Delta T_{s, s}$ of CC method and $T_{s, e}$ of CTD method applied for the stilbene scintillator (Figure 6A and 6B) and the EJ-301 liquid scintillator (Figure 6C and 6D). In case of stilbene detector with the CC method, the FOM value increases until $\Delta T_{s, s}$ becomes 36 ns and becomes saturated (Figure 6A). In the CTD method, the FOM value starts to show saturation after $T_{s, e}$ is $34 \mathrm{~ns}$ (Figure 6B). $\Delta T_{s, s}$ and $T_{s, e}$ values with the highest FOM were achieved at $36 \mathrm{~ns}$ and $34 \mathrm{~ns}$, respectively for the stilbene scintillator detector. In case of EJ-301, optimized $\Delta T_{s, s}$ and $T_{s, e}$ were $32 \mathrm{~ns}$ and $30 \mathrm{~ns}$, respectively. When a time parameter $\left(\Delta T_{s, s}\right.$ or $\left.T_{s, e}\right)$ of 34 ns or more is applied to the EJ-301 liquid scintillator, the PSD parameters were not able to be calculated for many signal pulses as one can tell from a typical pulse shape shown in Figure 3, making it impossible to calculate FOM values either. In this regard, time parameters $\left(\Delta T_{s, s}\right.$ or $\left.T_{s, e}\right)$ tested here were kept under $32 \mathrm{~ns}$ for the EJ-301 liquid scintillator detector and $42 \mathrm{~ns}$ for the stilbene scintillator de- tector. In addition, faster processing of the signal that also takes up less memory is always desired, therefore, PSD processing of pulses with unnecessarily long-time parameters would be meaningless. Optimized $\Delta T_{s, s}$ and $T_{s, e}$ for EJ-301 was shown somewhat smaller than those for stilbene scintillator, because the decay time of EJ-301 liquid scintillator and the time response value of R7724 PMT were shorter.

The result of the PSD experiment using the CC method and the CTD method, obtained with optimized time variables, is shown in Figures 7 and 8. Two-dimensional PSD plot at full energy range and the distribution of PSD parameters were obtained by measuring ${ }^{252} \mathrm{Cf}$ and ${ }^{137} \mathrm{Cs}$ sources. One can observe high-counts in the 300 to $500 \mathrm{keVee}$ region of gamma-ray area of PSD plots (Figures 7 and 8). This highcount region corresponds to the Compton edge and the Compton continua regions of ${ }^{137} \mathrm{Cs}$. Comparing high count regions of ${ }^{137} \mathrm{Cs}$ obtained by two different PSD methods applied on the same type of scintillators show similarities with 

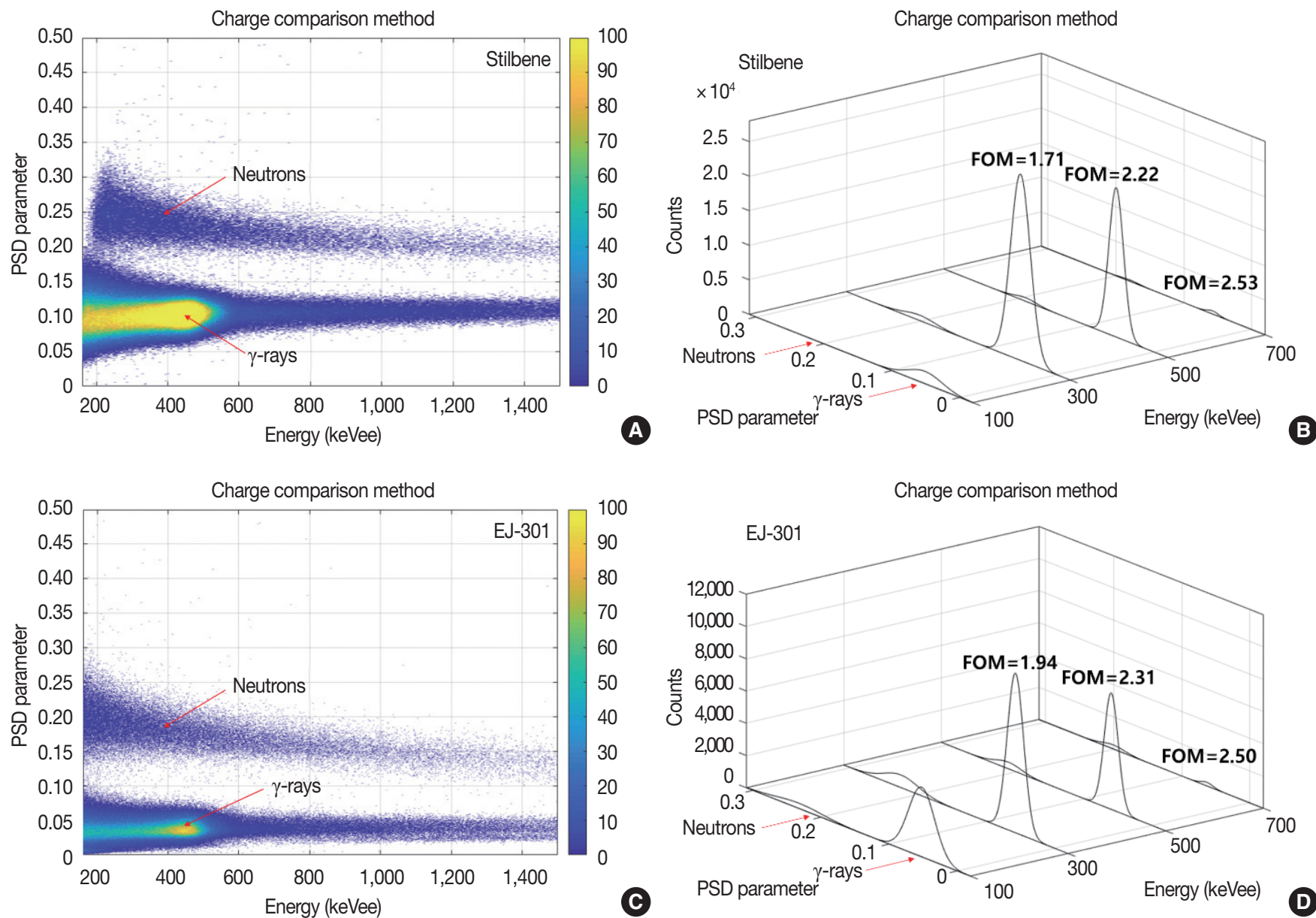

Fig. 7. (A) PSD plot at full energy range and (B) distribution of PSD parameter at various energies obtained with the CC method in the optimized set-up, using the stillbene scintillator measuring ${ }^{252} \mathrm{Cf}$ and ${ }^{137} \mathrm{Cs}$ sources. (C) PSD plot at full energy range and (D) distribution of PSD parameter at various energies obtained with the CC method in the optimized set-up, using the EJ-301 liquid scintillator measuring ${ }^{252} \mathrm{Cf}$ and ${ }^{137} \mathrm{Cs}$ sources.

each other, which buttresses compatibility and reliability of both PSD methods in relationship with each other. Optimized PSD variables that present the highest FOM value using the CC method and the CTD method are summarized in Table 2. FOM values, higher than 1.5, were achieved for all cases with these optimized PSD variables.

In Figures 7B, 7D, 8B, and 8D, FOM values and profiles of neutron/gamma-ray separation at different energy regions (300 \pm 100 keVee, $500 \pm 100$ keVee, $700 \pm 100 \mathrm{keVee}$ ) are shown. At $300 \mathrm{keVee}, 500 \mathrm{keVee}$, and $700 \mathrm{keVee}$ regions, FOM values were shown to be greater than the FOM values in the full energy range. Figure 9 shows the FOM value depending on $\Delta T_{s, s}$ of CC method and $T_{s, e}$ of CTD method applied for the stilbene scintillator (Figure 9A and 9B) and the EJ-301 liquid scintillator (Figure 9C and 9D) at different energy regions. As energy value increases, the size and the duration of the pulse increases. Therefore, the larger the energy value of the re- gion, the FOM value tends to be higher with larger time variables. However, the FOM value was maintained above 1.5 even with the optimized PSD variables for the full energy range shown in Table 2. This shows that we can obtain a desirable FOM value which can clearly distinguish neutrons from gamma rays regardless of the energy step using the optimized PSD parameters in Table 2. This is in line with our goal of making a dual-particle imager.

In this study, EJ-301 liquid scintillator showed slightly better FOM values than stilbene, however, one cannot simply conclude the EJ-301 liquid scintillator is a better detector for PSD methods, as we did not bring other factors that can be still optimized - PMTs used for each detector, detector efficiency, handling issues etc. - into our consideration for neutron detection in a complex radiation field. Furthermore, when utilizing these scintillators for a dual-particle imager, a detection efficiency as well as PSD performance is a major 

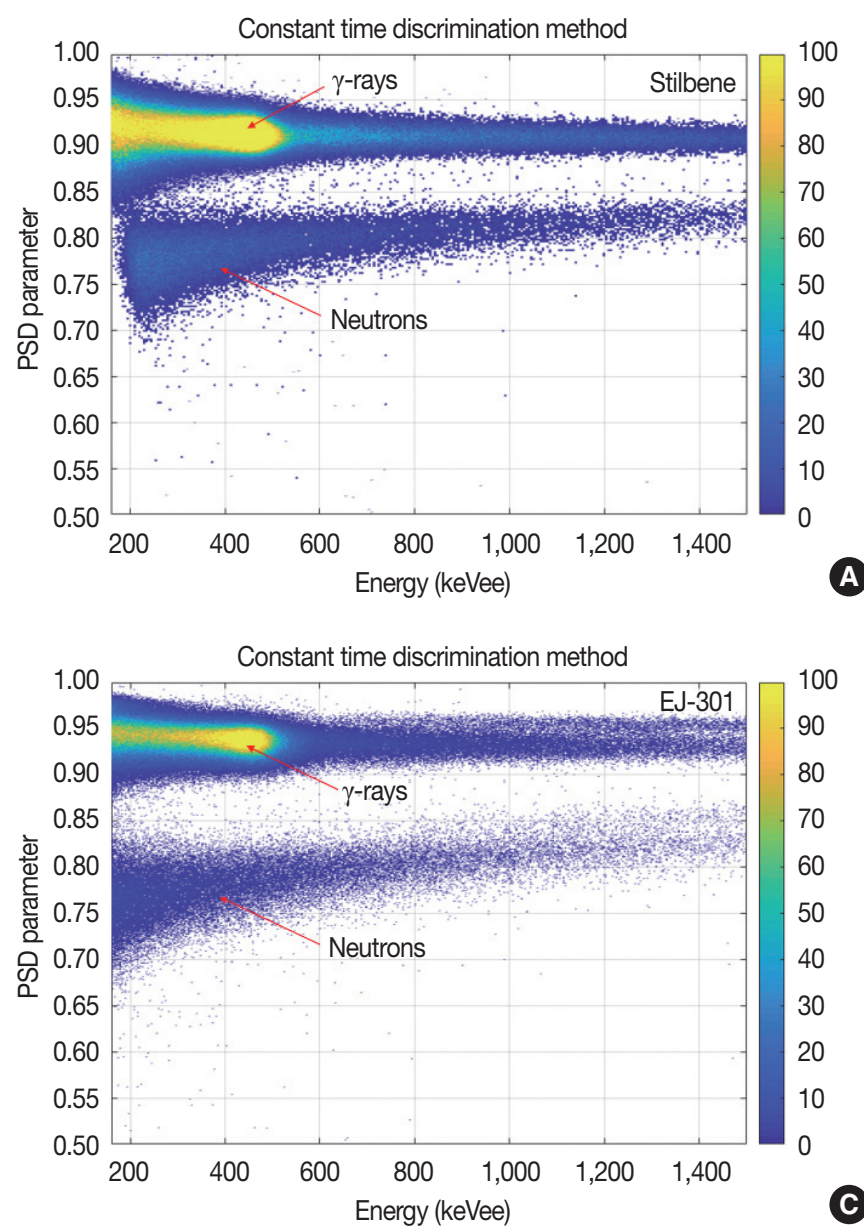

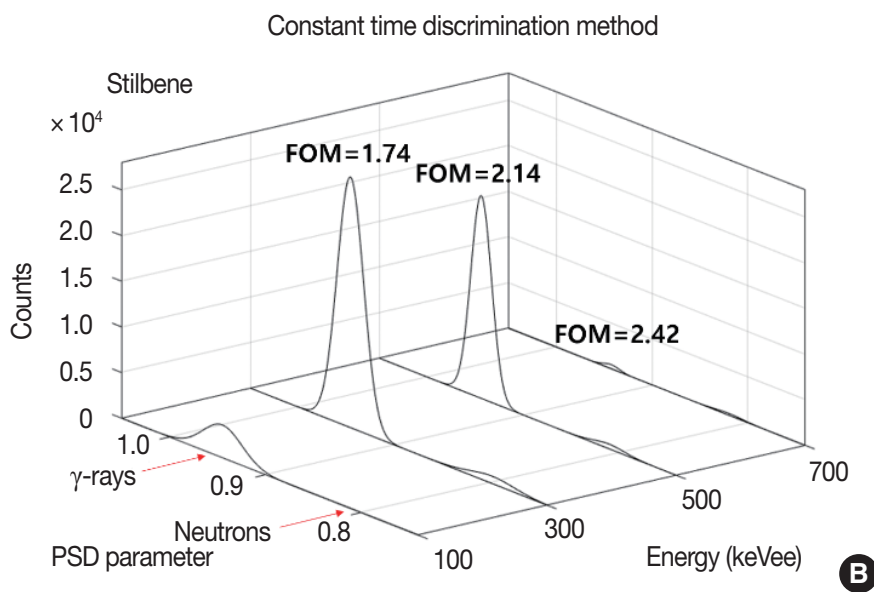

Constant time discrimination method

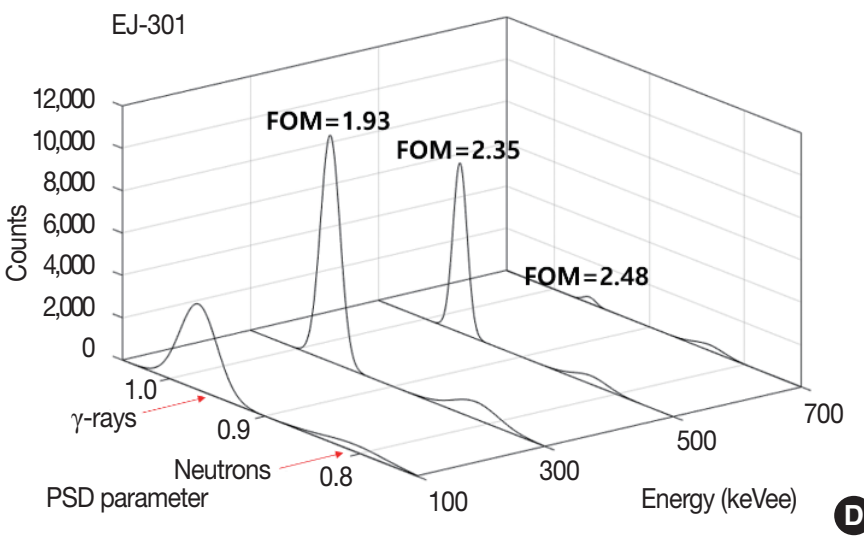

Fig. 8. (A) PSD plot at full energy range and (B) distribution of PSD parameter at various energies obtained with the CTD method in the optimized set-up, using the stilbene scintillator measuring ${ }^{252} \mathrm{Cf}$ and ${ }^{137} \mathrm{Cs}$ sources. (C) PSD plot at full energy range and (D) distribution of PSD parameter at various energies obtained with the CTD method in the optimized set-up, using the EJ-301 liquid scintillator measuring ${ }^{252} \mathrm{Cf}$ and ${ }^{137} \mathrm{Cs}$ sources.

Table 2. Optimized Threshold and Time Variables Conditions for PSD at the Full Energy Range

\begin{tabular}{llcrl}
\hline Scintillator & \multicolumn{1}{c}{$\begin{array}{c}\text { PSD } \\
\text { Method }\end{array}$} & $\begin{array}{c}\text { Thresholds for } T_{t, s}, T_{t, e} \\
\text { (relative to the } \\
\text { maximum amplitude) }\end{array}$ & $\begin{array}{c}\text { Optimal time } \\
\text { variable }\end{array}$ & $\begin{array}{c}\text { FOM } \\
\text { value }\end{array}$ \\
\hline \multirow{2}{*}{ Stilbene } & CC method & $1.5 \%$ & $\Delta T_{s, s}=36 \mathrm{~ns}$ & 1.70 \\
& CTD & $1.5 \%$ & $T_{s, e}=34 \mathrm{~ns}$ & 1.70 \\
EJ-301 & CC method & $0.5 \%$ & $\Delta T_{s, s}=32 \mathrm{~ns}$ & 1.79 \\
& CTD & $0.5 \%$ & $T_{s, e}=30 \mathrm{~ns}$ & 1.79 \\
\hline
\end{tabular}

consideration. In this regard, stilbene detector could have an advantage of being solid-state over the EJ-301 liquid scintillator, to be implemented in a dual-particle imager.

\section{Conclusion}

As an initiative attempt to implement the digital PSD technique on the stilbene scintillator detector and the EJ-301 liq- uid scintillator detector using a high-band width digitizer DT5730, this study was performed to deduce optimized PSD variables for the CC method and the CTD method using the two types of scintillator detectors. Optimized PSD variables was selected by comparing FOM values depending on thresholds, time interval ( $\Delta T_{s, s}$ for CC) and constant time $\left(T_{s, e}\right.$ for CTD) at the full energy range. We obtained FOM values higher than 1.5, with both scintillator detectors using the CC method and the CTD method Especially, the high FOM value was obtained even at 200-500 keVee which is the Compton continua region of ${ }^{137} \mathrm{Cs}$. The stilbene scintillator detector and the EJ-301 liquid scintillator detector did not show significant differences in its PSD performance between the CC method and the CTD method. Better PSD performance for the stilbene scintillator and the EJ-301 scintillator were observed by adjusting time interval and constant time values to a certain range at each energy step and the full energy range. 


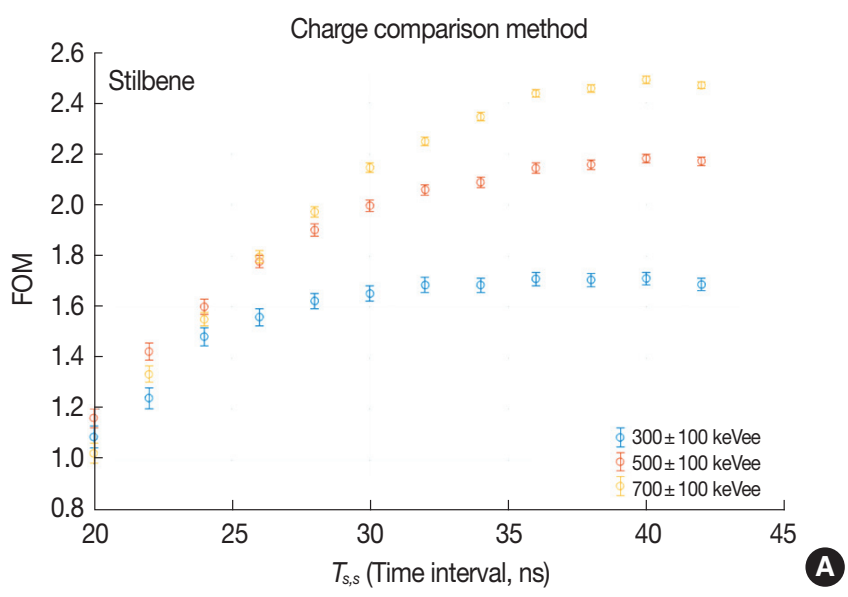

Charge comparison method

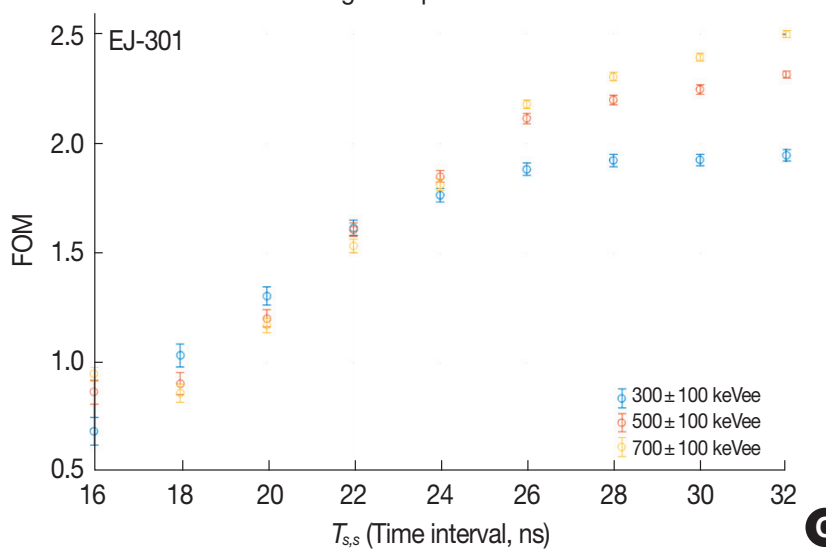

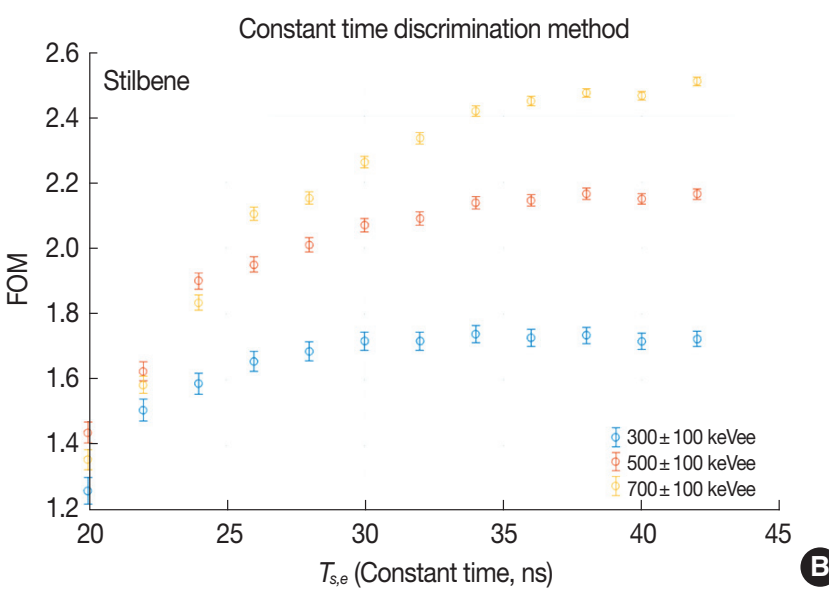

Constant time discrimination method

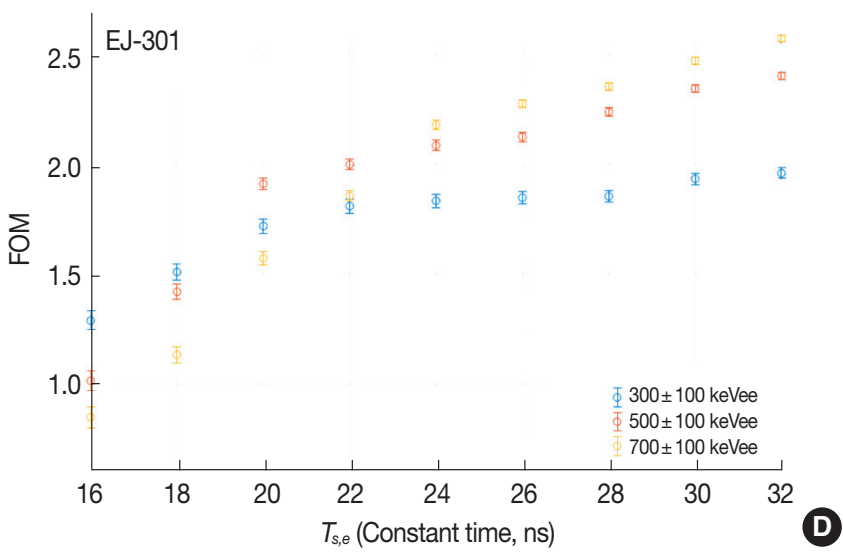

Fig. 9. At each energy steps, FOM values calculated for (A) the CC method and (B) the CTD method of the stillbene scintillator and (C) the CC method and (D) the CTD method of the EJ-301 liquid scintillator applying various time parameter $\left(\Delta T_{s, s}\right.$ and $\left.T_{s, e}\right)$ and threshold conditions.

Thresholds to determine the beginning and the ending points to process the signal is also an important parameter to consider in digital PSD technique. The FOM difference of more than 0.5 was observed in some cases, by changing threshold values from $1.5 \%$ to $0.5 \%$ of the maximum amplitude (Figure 6C and 6D). This shows that PSD performance can be substantially affected by the determination of the threshold. Optimized variables for two digital PSD methods can provide reference values for developing a fast neutron/ gamma-ray dual-particle imager with the stilbene scintillator and the EJ-301 liquid scintillator as feasibility discussed in one of previous studies [10-12].

\section{Acknowledgements}

This work was partially supported by the Nuclear Safety Research Program through the Korea Foundation Of Nuclear Safety (KOFONS), granted financial resources from the $\mathrm{Nu}-$ clear Safety and Security Commission (NSSC), Republic of Korea (No. 1403024) and partially by the National Research Foundation of South Korea (NRF-2018R1D1A1A02048400).

\section{References}

1. Knoll GF, Radiation detection and measurement. 4th Ed. New york. John Wiley \& Sons. 2010.

2. Brooks F. Development of organic scintillators, Nucl. Instrum. Methods. 1979;162(1-3):477-505.

3. Pozzi S, Clarke S, Flaska M, Peerani P. Pulse-height distributions of neutron and gamma rays from plutonium-oxide samples. Nucl. Instrum. Methods Phys. Res., Sect. A. 2009;608(2):310-315.

4. Carman L, Zaitseva N, Martinez HP, Rupert B, Pawelczak I, Glenn A, Mulcahy H, Leif R, Lewis K, Payne S. The effect of material purity on the optical and scintillation properties of solutiongrown trans-stilbene crystals. J. Cryst. Growth. 2013;368:56-61.

5. Plischke P, Schröder V, Scobel W, Wilde L, Bormann M. An electronic circuit for pulse shape discrimination in organic scintilla- 
tors. Nucl. Instrum. Methods. 1976;136(3):579-583.

6. Perkins L, Scott MC. The application of pulse shape discrimination in NE 213 to neutron spectrometry. Nucl. Instrum. Methods. 1979;166(3):451-464.

7. Söderström PA, Nyberg J, Wolters R. Digital pulse-shape discrimination of fast neutrons and $\gamma$ rays. Nucl. Instrum. Methods Phys. Res. Sect. A. 2008;594(1):79-89.

8. Kaschuck Y, Esposito B. Neutron/ $\gamma$-ray digital pulse shape discrimination with organic scintillators. Nucl. Instrum. Methods Phys. Res. Sect. A. 2005;551(2-3):420-428.

9. Bell ZW. Tests on a digital neutron-gamma pulse shape discriminator with NE213. Nucl. Instrum. Methods Phys. Res. 1981;188 (1):105-109.

10. Kim HS, Choi HY, Lee G, Ye SJ, Smith MB, Kim G. A Monte Carlo simulation study for the gamma-ray/neutron dual-particle imager using rotational modulation collimator (RMC). J. Radiol. Prot. 2018;38(1):299.

11. Kim HS, Smith MB, Koslowsky MR, Kwak SH, Ye SJ, Kim G. Characterization of a CLYC detector and validation of the Monte Carlo simulation by measurement experiments. J. Radiol. Prot. Res. 2017;42(1):48-55.

12. Kim HS, Ye SJ, Shin Y, Lee G, Kim G. Radiation imaging with a rotational modulation collimator (RMC) coupled to a $\mathrm{Cs}_{2} \mathrm{LiYCl}_{6}$ : Ce: (CLYC) detector. J. Korean Phys. Soc. 2016;69(11):1644-1650.
13. Bourne M, Clarke S, Adamowicz N, Pozzi S, Zaitseva N, Carman L. Neutron detection in a high-gamma field using solutiongrown stilbene. Nucl. Instrum. Methods Phys. Res. Sect. A. 2016;806:348-355.

14. Cieślak M, Gamage KA, Glover R. Pulse shape discrimination characteristics of stilbene crystal, pure and 6Li loaded plastic scintillators for a high resolution coded-aperture neutron imager. J. Instrum. 2017;12:P07023.

15. Feng PL, Villone J, Hattar K, Mrowka S, Wong BM, Allendorf MD, Doty FP. Spectral- and pulse-shape discrimination in tripletharvesting plastic scintillators. IEEE Trans. Nucl. Sci. 2012;59(6): 3312-3319.

16. Lang R, Masson D, Pienaar J, Röttger S. Improved pulse shape discrimination in EJ-301 liquid scintillators. Nucl. Instrum. Methods Phys. Res. Sect. A. 2017;856:26-31.

17. Winyard R, Lutkin J, McBeth G. Pulse shape discrimination in inorganic and organic scintillators. I. Nucl. Instrum. Methods. 1971;95(1):141-153.

18. http://www.eljentechnology.com, May 2018.

19. https://www.hamamatsu.com, September 2018.

20. Zaitseva N, Glenn A, Carman L, Martinez HP, Hatarik R, Klapper $\mathrm{H}$, Payne S. Scintillation properties of solution-grown trans-stilbene single crystals. Nucl. Instrum. Methods Phys. Res. Sect. A. 2015;789: 8-15. 